\title{
Práticas preventivas realizadas por Técnicos em Saúde Bucal: um estudo qualitativo
}

\author{
Tânia Harumi Uchida*, Raquel Sano Suga Terada**, Lívia Maria Andaló Tenuta***, Mitsue
} Fujimaki****

\author{
Mestranda, Programa de Pós-Graduação em \\ Odontologia Integrada da Universidade \\ Estadual de Maringá \\ ** Professora Associada da Universidade \\ Estadual de Maringá \\ *** $\quad$ Professora Associada da Universidade \\ Estadual de Campinas (Faculdade de \\ Odontologia de Piracicaba) \\ ***** Professora Adjunta da Universidade Estadual \\ de Maringá
}

\section{RESUMO}

A cárie dentária é uma doença comum que pode ser totalmente controlada. Entretanto, a percepção de técnicos em saúde bucal (TSBs) quanto sua prevenção ainda é pouco explorada. O objetivo desse estudo foi identificar a percepção dos TSBs com relação aos fatores facilitadores para adoção de práticas de prevenção no controle da cárie dentária. Participaram desta pesquisa 10 TSBs pertencentes à $15^{\mathrm{a}}$ Regional de Saúde do Paraná. As entrevistas semiestruturadas foram realizadas face a face e gravadas. A transcrição das gravações foi realizada de forma manual e avaliada segundo a análise de conteúdo proposta por Bardin, utilizando-se o software Visual Qualitative Data Analysis 7.0. Os resultados apontaram 12 fatores facilitadores para a prevenção da cárie, dentre eles: capacitação profissional; consciência, satisfação e motivação em realizar procedimentos preventivos e trabalho em equipe. Além disso, a habilidade de trabalhar com educação em saúde e com educação permanente foram fatores relevantes destacados nas entrevistas. De modo geral, os fatores facilitadores quanto a adoção de práticas de prevenção no controle da cárie dentária, identificados na percepção dos TSBs foram a consciência de realizar a prevenção na sua prática diária; a habilidade de comunicação para realizar educação em saúde e a satisfação do profissional em trabalhar com prevenção. Todos esses fatores relatados parecem ser necessários para o desempenho adequado da função dos TSBs, superando o modelo biologicista e avançando no entendimento do processo saúde-doença, a fim de implementar estratégias para o controle da cárie dentária na população.

Descritores: Auxiliares em Odontologia. Pesquisa Qualitativa. Técnico em Saúde Bucal.

\section{INTRODUÇÃO}

A cárie dentária é um importante problema de saúde pública em todo o mundo, devido à dor, ao sofrimento causados aos indivíduos, ao alto custo do seu tratamento e ao impacto na qualidade de vida ${ }^{1}$. Em grande parte dos países em desenvolvimento, $70 \%$ das crianças possuem ou possuíram alguma experiência de cárie, sendo na maior parte dos casos lesões não tratadas ${ }^{2}$. No último levantamento epidemiológico nacional das doenças bucais conduzido pelo Ministério da Saúde (MS), no ano de 2010, apesar da expressiva diminuição do índice de cárie em diversas faixas etárias e do aumento do acesso 
aos serviços de saúde ${ }^{3,4}$, ficou evidenciado que a população continua a perder seus dentes ao longo da vida ${ }^{5,6}$. Assim, o trabalho com promoção de saúde ainda é um grande desafio tanto na abordagem individual quanto coletiva, já que a cárie dentária é uma doença crônica passível de ser controlada a partir de medidas educativas.

Neste contexto, dentre os membros da equipe de saúde bucal, os técnicos e auxiliares em saúde bucal (TSBs e ASBs) exercem papel fundamental no cuidado à saúde, já que estabelecem o principal elo com a população e atuam diretamente na promoção da saúde e na prevenção das doenças bucais ${ }^{7}$. Leavell \& Clark $^{8}$, descrevem a prevenção em saúde como uma ação antecipada, baseada no conhecimento da história natural a fim de tornar improvável o progresso posterior da doença. Dentro do contexto da prevenção, a educação assume papel relevante, pois o indivíduo deixa de ser passivo aos programas de saúde bucal e passa a atuar como colaborador ${ }^{9}$. Em especial, a educação em saúde bucal é importante por permitir ao indivíduo o acesso ao conhecimento sobre as doenças bucais que podem acometê-lo e seu curso natural, bem como sobre a melhor forma para sua prevenção ${ }^{10}$.

O trabalho articulado e sinérgico da equipe de saúde bucal é necessário para a construção e consolidação das redes de atenção à saúde ${ }^{11}$, principal diretriz para o funcionamento dos serviços de saúde no país. Assim, a consciência de seu papel e de sua responsabilidade na prevenção das doenças e promoção de saúde são pontos fundamentais para que a força de trabalho da equipe auxiliar odontológica cumpra adequadamente a sua função. Entretanto, na literatura pouco se sabe sobre a percepção destes em relação aos fatores de influência para a prevenção da cárie dentária. A maioria dos estudos realizados na
América Latina são investigações epidemiológicas ou de natureza quantitativa, demonstrando que a percepção preventiva de TSBs é pouco procurado e discutido. Como resultado, as informações sobre o significado e a intencionalidade inerente às percepções e atitudes preventivas, ou sua ausência, ainda é escassa.

Esses profissionais auxiliares compõem a equipe odontológica juntamente com o cirurgião-dentista (CD) e realizam atividades necessárias à prestação de cuidados no âmbito da promoção, prevenção e recuperação da saúde bucal ${ }^{12}$. Atuam nas unidades e serviços de saúde, conveniados ou não ao Sistema Único de Saúde (SUS), estando em expansão sua inserção em Equipes de Saúde da Família (ESF). Segundo dados do MS, 23.150 Equipes de Saúde Bucal (ESB) encontram-se espalhadas em 4.971 municípios brasileiros, o que corresponde a uma cobertura de 89,2\% dos municípios, onde a equipe auxiliar está trabalhando em conjunto com o CD e a equipe multiprofissional da ESF de acordo com o Conselho Federal de Odontologia $(\mathrm{CFO})^{13}$. O Paraná conta atualmente com 1.199 ESB, sendo 823 na modalidade I e 376 na modalidade II, o que corresponde a 39,6\% de cobertura no Estado, segundo a Secretária de Saúde do Estado do Paraná $^{14}$.

No Brasil, a primeira proposta para regulamentar o pessoal auxiliar foi o Parecer 460 de 1975 do antigo Conselho Federal de Educação $^{15}$. Diversos movimentos democráticos a favor da utilização de pessoal auxiliar na odontologia aconteceram na década de 1980, em contraposição a interesses corporativos que se contrapunham a essa utilização. Assim, de acordo com Narvai e Frazão ${ }^{16}$, a profissionalização do Técnico em Saúde Bucal (TSB), bem como o rol de atribuições permaneceram comprometidos, durante a longa história de consolidação dessa ocupa- 
ção no Brasil. Em 1984, o CFO reconhece esses profissionais, disciplinando o exercício dos mesmos ${ }^{17}$. Mas somente no ano de 2008, houve a publicação da Lei Federal n ${ }^{\circ} 11.889$, descrevendo as atribuições e as restrições dos TSB no âmbito da saúde bucal ${ }^{7}$.

De acordo com Pezzato ${ }^{18}$, o SUS tem sido o principal empregador do TSB, do ASB e do CD. Segundo o MS, o SUS emprega $30 \%$ dos dentistas do país. Em 2002, este número era de 40.205 e, atualmente, encontra-se 63.584 profissionais atuando na rede pública, o que significa um aumento de $49 \%$, segundo o $\mathrm{CFO}^{13}$. Da mesma maneira, é provável que a equipe auxiliar tenha aumentado em proporção semelhante. Logo, estamos diante de um grande contingente trabalhando no cuidado da saúde bucal, cujo exercício profissional pode interferir diretamente na qualidade de vida da população.

Assim, o objetivo desse estudo foi identificar a percepção dos TSBs com relação aos fatores facilitadores para adoção de práticas de prevenção no controle da cárie dentária.

\section{METODOLOGIA}

O estudo caracterizou-se como uma pesquisa qualitativa com o objetivo de explorar as diferentes percepções dos TSBs em relação à prevenção da cárie dentária. Foram realizadas entrevistas semiestruturadas com os TSBs pertencentes a $15^{\text {a }}$ Regional de Saúde do Paraná. As entrevistas foram gravadas, transcritas e analisadas segundo o método de análise de conteúdo proposta por $\operatorname{Bardin}^{19}$.

\section{Sujeitos da pesquisa}

A amostra foi constituída por 10 TSBs, atuantes nas Unidades Básicas de Saúde (UBSs) pertencentes à $15^{\text {a }}$ Regional de Saúde do Paraná, indicados pelos coordena- dores de saúde bucal dos respectivos municípios por exercerem sua função de maneira adequada. Ao coordenador de saúde bucal foi solicitado que contactasse os coordenadores municipais de saúde bucal, para que indicassem os TSBs que desempenhassem de maneira satisfatória as suas funções.

Todos os TSBs foram inicialmente contactados por telefone ou e-mail. Destes profissionais, todas eram mulheres, possuindo idade média de 40 anos e média de 17 anos de serviço prestado ao SUS. As entrevistas foram realizadas mediante assinatura do Termo de Consentimento Livre e Esclarecido e de forma presencial, "face a face" e conduzidas por um roteiro. A participação foi voluntária e os participantes podiam retirar a sua autorização em qualquer momento da pesquisa. O projeto foi submetido ao Comitê de Ética em Pesquisa Envolvendo Seres Humanos (COPEP) da Universidade Estadual de Maringá (UEM) e aprovado na reunião do dia 17 de fevereiro de 2014, sob o CAAE $\mathrm{N}^{\circ}$ 23100213.3.0000.0104.

\section{Roteiro}

A partir de revisão de literatura foram analisados os principais artigos sobre a temática abordada. Inicialmente foi elaborado um roteiro, que foi melhorado com o auxílio de pesquisadores com experiência em análise qualitativa. Foram realizadas três reuniões para discussão e realização das alterações sugeridas, de maneira a evitar questões ou abordagens tendenciosas no roteiro final.

O roteiro foi composto por tópicos que eram abordados durante a entrevista, como por exemplo a visão do TSB quanto as práticas preventivas realizadas nas UBSs, relacionamento com a equipe de saúde tratando-se de prevenção, as experiências sobre medidas preventivas durante o curso de 
formação, como as ações coletivas influenciam a prevenção de cárie na UBS e se havia incentivos financeiros para a prevenção em saúde bucal.

\section{A entrevista}

Todas as entrevistas foram realizadas por uma estudante do $5^{\circ}$ ano do Curso de Odontologia na UEM, treinada e capacitada para tal. Não houve nenhum vínculo de interesse ou de trabalho entre ambas as partes com o objetivo de manter a imparcialidade durante as entrevistas e análise dos dados. A confidencialidade dos dados foi mantida, não identificando as respostas antes da análise e tomando todos os cuidados necessários para não expor detalhes que pudessem identificar os entrevistados.

As entrevistas foram realizadas até o ponto de saturação dos dados e tiveram de 20 a 40 minutos de duração. Um gravador portátil e o software Camtasia Studio ${ }^{\circledR}$ v. 7.1.1 build 1785 (TechSmid, Okemos, MI, EUA) foram utilizados para o registro das entrevistas. A transcrição das mesmas foi realizada manualmente pela entrevistadora para possibilitar uma leitura longitudinal que permitiu editá-las, sem alterá-las. A dinâmica da entrevista seguiu um roteiro de perguntas, porém a entrevistadora pôde conduzir o diálogo a fim de obter as informações desejadas sem fugir da proposta ou mesmo desviar do objetivo da pesquisa. Dentro dessa abordagem, procurou-se dar liberdade para que o entrevistado falasse o que julgasse importante.

\section{Procedimentos de Análise}

Ao término de cada entrevista, a entrevistadora responsável realizou manualmente a análise das falas, bem como das reações e fez anotações individualizadas, na tentativa de mergulhar no universo e percepções dos TSBs. Além disso, as unidades de registro foram pontuadas.

Ao final das entrevistas, as transcrições foram analisadas manualmente, com o objetivo de certificação da saturação dos dados. No momento em que não foi percebida nenhuma nova unidade de registro, foram realizadas mais três entrevistas para a certificação da saturação dos dados.

Em seguida, todas as transcrições foram analisadas no software Visual Qualitative Data Analysis 7.0 (Atlas.ti ${ }^{\circledR}$ Scientific Software Development, Berlim, Alemanha). Cada entrevista foi analisada três vezes.

Para guiar a análise, foram utilizadas como base 13 categorias (códigos) de dados que emergiram da análise de um referencial teórico composto de cinco artigos provenientes de uma revisão sistemática do mesmo tema da pesquisa ${ }^{20}$.

As expressões chaves e as ideias centrais, designadas como unidades de registro, foram identificadas de acordo com o contexto inserido, designado como unidade de contexto.

As unidades de registro foram agrupadas de acordo com o mesmo sentido, sentido equivalente ou sentido complementar em categorias, que por sua vez, foram agrupadas em unidades maiores, designadas famílias $^{20}$.

A frequência dessas categorias permitiu a ordenação dos achados e uma abordagem numérica descritiva do material empírico.

O quadro 1 apresenta as categorias de dados agrupados conforme sua família, a referência bibliográfica de cada código e sua definição constitutiva, que se refere ao significado funcional. 
Quadro 1 - Organização e definição das categorias de dados (códigos)

\begin{tabular}{|c|c|c|c|}
\hline Família & Categorias de dados & Referências & Definição Constitutiva \\
\hline \multirow[t]{11}{*}{ PROFISSIONAL } & Satisfação & Holloaway, 1994 & $\begin{array}{l}\text { Satisfação em trabalhar com } \\
\text { prevenção ou desejo de } \\
\text { melhorar. }\end{array}$ \\
\hline & Capacitação & Sbaraini, 2012 & $\begin{array}{c}\text { Atualização constante do } \\
\text { profissional. }\end{array}$ \\
\hline & $\begin{array}{l}\text { Procedimentos } \\
\text { Preventivos }\end{array}$ & Holloaway, 1994 & $\begin{array}{c}\text { Realização dos } \\
\text { procedimentos preventivos, } \\
\text { entendendo os seus } \\
\text { benefícios. }\end{array}$ \\
\hline & $\begin{array}{l}\text { Consciência } \\
\text { Preventiva }\end{array}$ & $\begin{array}{c}\text { Holloaway, } 1994 \\
\text { Threlfall, } 2007 \\
\text { Nettleton, } 1989 \\
\text { Sbaraini, } 2012 \\
\end{array}$ & $\begin{array}{c}\text { Consciência preventiva e } \\
\text { visão preventiva implícita em } \\
\text { atitudes. }\end{array}$ \\
\hline & $\begin{array}{l}\text { Constrangimento do } \\
\text { Paciente }\end{array}$ & Nettleton, 1989 & $\begin{array}{l}\text { Constrangimento do paciente } \\
\text { ao ser orientado ou corrigido } \\
\text { pelo profissional. }\end{array}$ \\
\hline & $\begin{array}{c}\text { Comunição e Educação } \\
\text { em Saúde }\end{array}$ & $\begin{array}{l}\text { Threfall, } 2007 \\
\text { Sbaraini, } 2012\end{array}$ & $\begin{array}{c}\text { Experiências em prevenção e } \\
\text { comunicação e poder de } \\
\text { persuasão. }\end{array}$ \\
\hline & Educação em Saúde & $\begin{array}{l}\text { Threfall, } 2007 \\
\text { Sbaraini, } 2012\end{array}$ & $\begin{array}{c}\text { Experiências em prevenção e } \\
\text { comunicação e poder de } \\
\text { persuasão. }\end{array}$ \\
\hline & Trabalho em Equipe & $\begin{array}{c}\text { Threlfall, } 2007 \\
\text { Holloway, } 1994 \\
\text { Sbaraini, } 2012\end{array}$ & $\begin{array}{c}\text { Benefícios de se trabalhar em } \\
\text { equipe, principalmente com } \\
\text { TSBs. }\end{array}$ \\
\hline & Educação Permanente & & $\begin{array}{l}\text { Presença de campanhas para } \\
\text { educar a população quanto a } \\
\text { prevenção. }\end{array}$ \\
\hline & Saúde Pública & & $\begin{array}{l}\text { Facilidade de trabalhar com } \\
\text { prevenção devido a um tempo } \\
\text { reservado a educação em } \\
\text { saúde. }\end{array}$ \\
\hline & Suporte & & $\begin{array}{l}\text { Disponibilidade de materiais } \\
\text { para prevenção. }\end{array}$ \\
\hline \multirow[t]{3}{*}{ PACIENTE } & Motivação & $\begin{array}{l}\text { Gussy, } 2006 \\
\text { Threlfall, } 2007\end{array}$ & $\begin{array}{c}\text { Motivação dos pacientes em } \\
\text { empregar métodos } \\
\text { preventivos. }\end{array}$ \\
\hline & Consciência & $\begin{array}{c}\text { Threlfall, } 2007 \\
\text { Nettleton, } 1988 \\
\text { Gussy, } 2006\end{array}$ & $\begin{array}{l}\text { Consciência preventiva, } \\
\text { relativa à fatores culturais, } \\
\text { econômicos, sociais e } \\
\text { educacionais. }\end{array}$ \\
\hline & Idade & Gussy, 2006 & $\begin{array}{l}\text { Facilidade de se trabalhar } \\
\text { prevenção com crianças e } \\
\text { adolescentes. }\end{array}$ \\
\hline
\end{tabular}




\section{RESULTADOS}

As principais falas, obtidas das e 3, dentro de suas respectivas categorias de entrevistas, estão apresentadas nos quadros 2 dados.

Quadro 2 - Categorias e respectivas unidades de registro obtidas a partir das entrevistas dos TSBs sobre práticas de prevenção relacionadas ao profissional

\begin{tabular}{|c|c|}
\hline Categorias & Unidades de Registro \\
\hline Capacitação & $\begin{array}{l}\text { E6005: Sempre quando tem cursos pela prefeitura, estou fazendo, porque é } \\
\text { importante para mim, como profissional, e para o bom atendimento ao paciente. }\end{array}$ \\
\hline $\begin{array}{l}\text { Consciência } \\
\text { Preventiva }\end{array}$ & $\begin{array}{l}\text { E1001: [...] a prevenção é você orientar o paciente e poder pegar o jeito, ter } \\
\text { paciência com o paciente, fazer evidenciação de placa, explicar de novo. } \\
\text { E8008: [...] a prevenção é o início de tudo. }\end{array}$ \\
\hline $\begin{array}{l}\text { Educação em } \\
\text { Saúde }\end{array}$ & $\begin{array}{l}\text { E1003: [...] muitas vezes pensamos que não vai dar em nada, que vai demorar um } \\
\text { pouco para surgirem os efeitos, para dar algum resultado, mas aí a gente percebe } \\
\text { que as crianças quando chegam na fase adulta, estão com os dentes saudáveis [...] } \\
\text { E4004: [...] se você ensinar o paciente a escovar os dentes, faz a orientação sob a } \\
\text { escovação, quando ele retornar daqui seis meses ou um ano, a boca vai estar ótima. }\end{array}$ \\
\hline $\begin{array}{l}\text { Procedimentos } \\
\text { Preventivos }\end{array}$ & $\begin{array}{l}\text { E1001: A palestra é o início do tratamento e depois no mesmo dia é feito a } \\
\text { escovação, a orientação, o uso do fio dental e então fazemos a triagem. } \\
\text { E9009: [...] Nós fazemos as palestras e escovação supervisionada, utilizamos o } \\
\text { macro modelo, ou seja, ensinamos como escovar [...] }\end{array}$ \\
\hline $\begin{array}{l}\text { Educação } \\
\text { Permanente }\end{array}$ & $\begin{array}{l}\text { E7007: [...] a presença da universidade dentro das creches e das escolas daqui } \\
\text { ajudou e muito na orientação das crianças. Percebemos a melhora que elas } \\
\text { apresentaram, estão sendo mais bem orientadas, praticamente sabem tudo sobre } \\
\text { escovação. }\end{array}$ \\
\hline Satisfação & $\begin{array}{l}\text { E8008: [...] é muito importante que o paciente visualize, por meio da evidenciação } \\
\text { de placa, que os seus dentes estão sujos. A partir disso, eu consigo mostrar para } \\
\text { ele, que a escovação não está boa. E é esse paciente que eu vou sempre orientar, } \\
\text { ensinar como escovar, até os seus dentes não corarem mais e isso me traz muita } \\
\text { satisfação, porque eu vejo o quanto posso ajudá-lo. }\end{array}$ \\
\hline Saúde Pública & $\begin{array}{l}\text { E3003: [...] trabalhar nas creches e nas escolas com prevenção, fazendo por } \\
\text { exemplo palestras educativas, pode diminuir sim o indice de cárie. Por isso que é } \\
\text { importante que essa parte preventiva é realizada desde a creche ou as escolas. }\end{array}$ \\
\hline Suporte & $\begin{array}{l}\text { E6006: [...] a coordenadora de saúde bucal do município é uma dentista e ela sabe } \\
\text { da importância de realizar a prevenção. Então tudo que tiver no alcance dela para } \\
\text { fazer uma palestra, por exemplo, ela não mede esforços para nos ajudar. }\end{array}$ \\
\hline $\begin{array}{l}\text { Trabalho em } \\
\text { Equipe }\end{array}$ & $\begin{array}{l}\text { E3003: A nossa equipe é muito boa, a dentista sempre está envolvida nas palestras } \\
\text { que a gente faz, principalmente na semana da saúde bucal. As auxiliares também } \\
\text { são bem comprometidas. }\end{array}$ \\
\hline
\end{tabular}


Quadro 3 - Categorias e respectivas unidades de registro, obtidas a partir das entrevistas dos TSBs, sobre práticas de prevenção relacionadas ao paciente

\begin{tabular}{|c|l|}
\hline Categorias & \multicolumn{1}{|c|}{ Unidades de Registro } \\
\hline $\begin{array}{c}\text { Consciência } \\
\text { preventiva }\end{array}$ & $\begin{array}{l}\text { E1001: [...] o usuário que recebe as informações, as palestras, as escovações } \\
\text { supervisionadas e tudo mais, a gente percebe que surge um efeito muito bom na } \\
\text { forma com que ele passa a cuidar dos seus dentes e da saúde bucal [...] }\end{array}$ \\
\hline Idade & $\begin{array}{l}\text { E7007: [...] percebemos aqui no consultório, que quanto mais jovem é o paciente } \\
\text { mais fácil é trabalhar a prevenção com ele. Mas, a idade não deve ser um } \\
\text { impedimento para orientarmos os nossos pacientes, pelo contrário, quanto mais } \\
\text { dificuldade ele tem, por ter mais idade ou não, é mais um motivo para ensinarmos } \\
\text { a escovar os dentes e passar o fio dental. }\end{array}$ \\
\hline Motivação & $\begin{array}{l}\text { E9009: [...] a motivação influencia, porque a gente chega aqui no no no } \\
\text { "escovódromo" depois da palestra, as crianças já entram aqui dentro com uma } \\
\text { nova visão sobre a escovação [...] }\end{array}$ \\
\hline
\end{tabular}

\section{DISCUSSÃO}

Esse estudo identificou a visão de TSBs da $15^{\text {a }}$ Regional de Saúde do Paraná quanto à prevenção da cárie dentária. Sua inovação se deve ao emprego da metodologia qualitativa para pesquisar em profundidade os fatores que facilitam a sua prevenção. Os fatores facilitadores que se destacaram nas falas dos TSBs, foram a consciência preventiva, procedimentos preventivos e a educação em saúde.

Em outubro de 2014, segundo dados do $\mathrm{CFO}^{13}$, existiam cerca de 18.847 TSBs no Brasil, 1.531 TSBs no estado do Paraná e 119 TSBs pertencentes a $15^{a}$ Regional de Saúde do Paraná. Esses profissionais prestam serviços de saúde à população desenvolvendo atividades e tarefas consideradas de menor complexidade, definidas pela Lei ${ }^{\circ} 11.889$, de 24 de dezembro de $2008^{7}$, que regulamentou o exercício das profissões de TSB e ASB.

Consta no artigo $5^{\circ}$ da referida lei, que compete ao TSB, sempre sob a supervisão do $\mathrm{CD}$, as seguintes atividades relacionadas a prevenção: participar das ações educativas atuando na promoção da saúde e na prevenção das doenças bucais; ensinar técnicas de higiene bucal e realizar a prevenção das doenças bucais por meio da aplicação tópica do flúor (Brasil, 2008) ${ }^{7}$.

Dentro do contexto de atuação multiprofissional do SUS, os técnicos em saúde constituem um contingente expressivo da força de trabalho no setor. De maneira geral, o termo "técnico" refere-se ao indivíduo que possui certa habilidade e/ou conhecimento específico sobre determinado assunto $^{21}$. O que se nota no Brasil é uma oferta de técnicos aquém da demanda de mercado ${ }^{12}$. Uma pesquisa de acompanhamento de egressos de cursos técnicos em várias áreas, envolvendo 550 formados por uma escola técnica, mostrou que $69 \%$ estavam inseridos no mercado de trabalho em atividades que indicam relação estreita entre formação técnica e perfil do emprego. Segundo Kirschiner $^{22}$, a demanda por esse tipo de escola vem crescendo no Brasil, o que não deixa de ser um reconhecimento público de sua qualidade e de sua relevância social. Da mesma maneira, podemos incluir os TSBs como uma força de trabalho importante, porém ainda pequena, já que de acordo com o 
$\mathrm{CFO}^{13}$ estão registrados 18.847 TSBs e 261.604 CDs. Em comparação com outros países europeus, os Países Nórdicos possuem relativamente mais TSBs por dentista. No entanto, a Noruega tem relativamente menos TSBs por dentista do que os outros países nórdicos, e consideravelmente menos do que o Canadá, Japão e os Estados Unidos, onde há quase um TSB por dentista ${ }^{23,24}$.

Considerando a categoria consciência preventiva (quadro 2 ), verificou-se que existe a visão preventiva, que pode ser observada por meio de atitudes, como a realização e persistência na prática da educação em saúde, tendo o entendimento do processo educativo como sendo lento e progressivo e requer comprometimento e dedicação profissional ${ }^{26}$. Além disso, um entendimento mais amplo também é observado quando o TSB relaciona a manutenção da saúde bucal com a necessidade de trabalhar a prevenção, sendo a prevenção, o "início de tudo", podendo ser entendido como alicerce, prioridade, base do tratamento odontológico. Da mesma forma, a consciência preventiva foi trabalhada com os pacientes (quadro 3), de maneira que os mesmos pudessem cuidar de sua própria saúde. A partir do relato do TSB, percebe-se que houve interação favorável entre profissional da saúde e paciente, no desenvolvimento desta competência.

A maioria dos participantes do trabalho, ao assumir o papel de educador em saúde, demonstrou consciência preventiva. Entretanto, resultados de um estudo realizado pela American Dental Hygienists Association, em 2012, mostrou que nos Estados Unidos somente 14\% dos 6.723 TSBs entrevistados disseram que seu papel principal não era só clínico e sim voltado para a prevenção ${ }^{23}$. De acordo com Bosi e Affonso $^{27}$, a consciência preventiva demonstra postura ética e engajamento proativo da pessoa naquilo que ela se propõe a fazer. Ela é um determinante à prestação de prevenção e sua eficácia, sendo evidenciada também nos estudos de Sbaraini ${ }^{28}$, Threfall et al. ${ }^{29} \mathrm{e}$ Nettleton ${ }^{30}$. Num estudo qualitativo com CDs, a consciência preventiva foi o principal motivador profissional da prevenção, muitas vezes sendo justificada pelo seu positivo custo-benefício, pela incorporação de um papel de educador pelo odontólogo, que se preocupa não só com a boca ou os dentes, mas sim com o bem-estar e qualidade de vida de seu paciente ${ }^{31}$. Além disso, a consciência profissional é um importante fator de influência na prevenção e esteve presente de forma direta ou indireta em todas as pesquisas qualitativas sobre esse tema ${ }^{2,28,29}$. Considerando que as TSBs participantes deste estudo foram escolhidas em função de seu desempenho adequado, a consciência preventiva era esperada. Entretanto, este ainda é um grande desafio para o $\mathrm{CD}$ ao capacitar TSBs, despertando a consciência preventiva. Da mesma maneira, os TSBs devem ter o objetivo de privilegiar a prevenção na educação de seus pacientes.

Métodos preventivos e curativos têm sido empregados para a diminuição da prevalência da cárie dentária. Dentre os mesmos é importante destacar a fluoretação das águas e o desenvolvimento de projetos de educação em saúde. Um estudo realizado na Noruega mostrou que a atuação de TSBs em educação para saúde bucal, dando informações sobre dieta, higiene e flúor, resultou em $70 \%$ das crianças de até 5 anos e $66 \%$ dos jovens de até 12 anos sem nenhum dente cariado, tanto em crianças de alto ou baixo risco à cárie dentária ${ }^{30}$. A maioria dessas crianças eram atendidas somente por TSBs. Este trabalho mostrou que à medida que o número de TSBs cresceu, as opiniões e práticas de prevenção tornaram-se cada vez 
mais prevalentes entre as crianças. Da mesma forma, os estados norte-americanos reconhecem cada vez mais a necessidade de prestar serviços de prevenção, assim como cuidados bucais efetivos e de baixo custo para um número maior de americanos. Eles acreditam que entre 2010 a 2022 o número de dentistas diminuirá, enquanto o número de TSBs aumentará em $38 \%{ }^{32}$. No Brasil, verifica-se que os TSBs são pouco valorizados e ainda não desempenham todo o seu potencial de trabalho, sendo necessário o desenvolvimento do trabalho em equipe.

A prática da educação em saúde é fundamental para a modificação dos hábitos visando à saúde. Os recortes das entrevistas (quadro 2) permitem observar seus resultados positivos. Os TSBs mostraram que entendem que a educação em saúde é um fator facilitador para a prevenção, apontando resultados de ações práticas das atribuições da equipe de saúde bucal. Algumas habilidades foram apontadas por TSBs como sendo importantes para o trabalho com educação em saúde: motivação, persuasão e comunicação ${ }^{33,34}$. O TSB tem uma gama de funções específicas para o desenvolvimento das ações voltadas à prevenção de doenças e promoção de saúde, podendo, com seu espaço e funções respeitadas e estimuladas, proporcionar à população melhores condições de atendimento.

Zarifian $^{15}$, ao trabalhar a noção de competência em educação em saúde, com base em preceitos científicos, realça que ela se sustenta na responsabilidade e iniciativa; base de conhecimentos que viabiliza a consolidação e a transformação de práticas; e capacidade de liderar e mobilizar equipes em função de determinada ação. A competência em educação em saúde foi identificada por meio da busca de artifícios que facilite o entendimento das mensagens transferidas pelo profissional, reforçando o vínculo com os pacientes $^{29}$, enaltecendo a crença na educação em saúde como principal meio de melhorar a saúde bucal e a busca de alternativas mais realistas para mudança de hábitos alimentares e comportamentais.

Foi verificado na fala da TSB (quadro 2) o entendimento quanto à importância de realizar cursos de capacitação. Fica claro na fala que os cursos não são ofertados regularmente pelo sistema. Assim, pode-se perceber a necessidade das prefeituras promoverem cursos de capacitação para TSBs de forma permanente, já que existe essa demanda de capacitação do serviço.

A utilização dos auxiliares na odontologia nem sempre foi aceita pelos CDs brasileiros $^{35}$. Mas essa não é uma realidade apenas brasileira. Pesquisa realizada na Noruega mostrou que os dentistas não estão dispostos a delegar tarefas para os $\mathrm{TSBs}^{36}$. Os CDs que atuam em áreas com uma grande demanda de profissionais da odontologia alegam perda de rendimento ao delegar tarefas para TSB. Os dentistas mais velhos no setor público, quando comparados com dentistas mais jovens do setor privado, foram mais positivos à delegação, o que reflete a crescente confiança da experiência pessoal de competência dos TSBs ao longo do tempo. Infelizmente, o que vemos na prática é a valorização do trabalho individual.

$\mathrm{Na}$ área da saúde esse fato é corroborado pelo monopólio do saber, o que implica num processo de trabalho individual, hierarquizado e fragmentado nos serviços de saúde. Na medida em que há o predomínio desse tipo de trabalho, a formação das categorias auxiliares encontra-se renegada e praticamente sem incentivo. No caso da Odontologia, o CD permanece com o status e o pessoal auxiliar odontológico marginalizado historicamente ${ }^{18}$. 
Tradicionalmente, o CD vinha assumindo posição central no campo de trabalho da saúde bucal. Contudo, há uma convergência das discussões para a necessidade de se orientar a prática odontológica a partir dos princípios preventivos e de humanização, além do reconhecimento de que ações requeridas para essa prática incluem ações de competência do pessoal auxiliar de nível técnico ${ }^{37,38}$. Isso se torna imprescindível a partir da constatação da saúde bucal como campo de conhecimento e de atuação complexa que concebe a incorporação de profissionais técnicos $\mathrm{e}$ auxiliares como uma necessidade, principalmente nas atividades de promoção da saúde bucal $^{39}$ e, também, nas atividades clínicas $^{40}$ e na gestão do trabalho na saúde bucal, cumprindo com a efetividade do serviço.

TSBs e ASBs, aos quais se refere esta pesquisa, são profissionais de saúde que podem desempenhar plenamente suas funções com qualificação. Quando não existe uma proposta de integração e trabalho em equipe, pode haver dificuldades na consolidação da identidade destes profissionais, causando uma mistura de papéis na equipe, em que um acaba exercendo ou ocupando o espaço do outro, o que descaracteriza o trabalho em equipe. Essa dificuldade não foi constatada na fala da TSB entrevistada. Pode-se verificar o bom relacionamento entre os membros da equipe, compreendendo que o papel de cada um dentro da equipe é importante.

Desta forma, é a própria equipe que perde sua identidade dentro e fora da classe odontológica. Fortalece-se, assim, a manutenção de um saber odontológico que é monopólio de poucos e, consequentemente, a Saúde Bucal permanece um sonho, inacessível à maioria da população brasileira. A falta de formação do CD para transferir funções ao TSB, para trabalhar com este profissional no consultório foi identificada como elemento que pode se constituir em barreira para a utilização do TSB nas ações clinicas $^{37}$.

No trabalho realizado por Aguiar ${ }^{41}$, a falta de confiança do CD no TSB, sentimento vivenciado por este, foi também mencionado como barreira à atuação do TSB. Nesse sentido, somente a comunicação horizontal na experiência de trabalho entre esses profissionais pode desmistificar a visão de que os TSBs existem para tomar espaço dos $\mathrm{CDs}$, sendo pouca a experiência que os CDs têm no trabalho em conjunto com os TSBs e partindo da visão preconceituosa que muitos ainda têm dessa relação como uma invasão de espaço.

Não é estranho que os CDs tenham restrições em atribuir responsabilidades aos TSBs no cotidiano dos serviços. Por isso, a importância em também se conhecer como vem se dando a relação entre TSB e os demais profissionais da saúde e, ainda, o grau de autonomia que ele dispõe em uma equipe de saúde ${ }^{41}$.

Para os profissionais de saúde bucal, sua inserção em uma equipe multiprofissional, significa uma maneira nova de trabalhar e representa ao mesmo tempo um avanço significativo e um novo espaço de construção de práticas e relações, que possibilita reorientar o processo de trabalho e a própria inserção da saúde bucal nos serviços de saúde, vislumbrando-se a possibilidade de aumento de cobertura, maior alcance de medidas de caráter coletivo e maior efetividade na resposta às necessidades da população $^{42}$.

Para Narvai ${ }^{37}$, a não utilização de pessoal auxiliar significa uma ostentação, que não deve ser permitida por nenhuma sociedade, considerando um erro empregar 
um profissional com elevado padrão cientifico na execução de ações que não requeiram tal padrão. Segundo Machado ${ }^{43}$, a redução de tempo e movimentos na execução de procedimentos clínicos é um dos princípios básicos da racionalização do trabalho em Odontologia, permitindo o aumento da produtividade profissional com o trabalho em equipe, ou seja, com transmissão de funções para o pessoal auxiliar.

De acordo com Frazão et al. ${ }^{17}$, a utilização de pessoal auxiliar odontológico possibilita, quando sob certas condições de trabalho, tanto do ponto de vista da eficiência quanto da eficácia, o aumento da qualidade, da produtividade e do rendimento dos serviços prestados pelo CD. Os TSBs devem ser o ponto de entrada para os serviços odontológicos e, na Noruega, a grande maioria (79\%) dos TSBs pensam assim, mas apenas $21 \%$ dos dentistas prestam apoio a essa hipótese. Porém 60\%, desses mesmos dentistas concordaram que os TSBs poderiam fazer mais exames, triagem e tratamentos básicos, atualmente desempenhados por dentistas ${ }^{44}$.

Nos Estados Unidos os TSBs têm contribuído para a saúde bucal da população, juntamente com dentistas nos consultórios. Isto permitiu que muitas pessoas tivessem acesso a melhores cuidados de saúde bucal no mundo, ainda assim, milhões são incapazes de obter até mesmo o atendimento odontológico básico que precisam ${ }^{32}$. É válido aliviar a pressão sobre dentistas por deixar TSBs assumirem algumas das suas tarefas, permitindo se concentrar em procedimentos que só eles são treinados para executar ${ }^{36}$. Isso aumentaria a eficiência no serviço prestado ${ }^{45}$. Assim, verifica-se a necessidade de maior valorização desta categoria profissional, ainda pouco ouvida e incluída nas discussões da equipe de saúde bucal.
É de suma importância que mais trabalhos, com uma abordagem qualitativa, sejam feitos na área da Odontologia, envolvendo CDs e a equipe auxiliar. Futuras pesquisas são necessárias para que possam ser avaliados, no âmbito de uma discussão coletiva, fatores que influenciam na realização da prevenção por TSBs e ASBs, assim como discutir estratégias para a incorporação dos procedimentos preventivos no processo de trabalho, por meio da utilização de um grupo focal.

As entrevistas realizadas nessa pesquisa foram realizadas com TSBs atuantes no serviço público e que exerciam adequadamente suas funções. Apesar das entrevistas evidenciarem preocupação e interesse em realizar práticas de prevenção, percebe-se ainda uma limitação no entendimento mais aprofundado e no treinamento sobre esta temática. Assim, é possível que outras percepções ainda menos claras sobre a importância da prática prevenção possam ser observadas em um grupo de TSBs menos comprometido.

\section{CONSIDERAÇÕES FINAIS}

De modo geral, os fatores facilitadores quanto à adoção de práticas de prevenção no controle da cárie dentária identificados na percepção dos TSBs foram: (1) a consciência de realizar a prevenção na sua prática diária; (2) a habilidade de comunicação para realizar educação em saúde, (3) a satisfação do profissional em trabalhar com prevenção, a partir da identificação dos seus benefícios; (4) ter tempo e (5) recursos necessários para trabalhar com prevenção. Todos esses fatores relatados parecem ser necessários para $\mathrm{o}$ desempenho adequado da função dos TSBs, superando o modelo biologicista e avançando no entendimento do processo saúde-doença, a 
fim de implementar estratégias para o controle da cárie dentária na população.

\section{AGRADECIMENTOS}

Ao professor Léo Kriger, Coordenador Estadual de Saúde Bucal do Paraná, à CD Rosangela Raddi Pedreiro Forestiero, Diretora da Atenção Primária da $15^{\text {a }}$ Regional de Saúde do Paraná, ao CD Fabio Garcia Furlan, Coordenador de Saúde Bucal da $15^{\text {a }}$ Regional de Saúde do Paraná, a CD Letícia Padovez, Coordenadora de Saúde Bucal de Maringá e às Técnicas em Saúde Bucal da $15^{\text {a }}$ Regional de Saúde do Paraná.

\section{ABSTRACT \\ Preventive practices carried out by technicians in oral health: a qualitative study}

Dental caries is a common disease that can be fully controlled. However, the perception of oral health technicians (OHTs) and its prevention is still little explored. The aim of this study was to identify the perception of OHTs with regard to factors that facilitate the adoption of practices in preventing the control of dental caries, participated in this study 10 OHTs belonging to the $15^{\text {th }}$ Regional of Health of Paraná. The semi-structured interviews were conducted face to face and recorded. The transcript of the recordings was done manually and evaluated according to the content analysis proposed by Bardin, using the Visual Qualitative Data Analysis 7.0 software. The results showed 12 facilitating factors for caries prevention, including: professional training; awareness, satisfaction and motivation in performing preventive procedures and teamwork. In addition, the ability to work with health education and continuing education were relevant factors highlighted in the interviews. In general, the risk factors as the adoption of preventive practices in controlling dental caries, identified in the perception of OHTs were the consciousness of performing prevention in their daily practice; the communication skills to carry out health education and professional satisfaction in working with prevention. All of these reported factors appear to be necessary for the proper performance of the function of OHTs, surpassing biologicist model and advancing the understanding of the healthdisease, in order to implement strategies for the control of dental caries in the population.

Descriptors: Dental Caries. Qualitative Research. Oral Health Technician.

\section{REFERÊNCIAS}

1. Petersen PE. World Health Organization global policy for improvement of oral health - World Health Assembly 2007. Int Dent J. 2008;58(3):115-21.

2. Gussy MG, Waters E, Kilpatrick NM. A qualitative study exploring barriers to a model of shared care for pre-school children's oral health. Br Dent J. 2006; 201(3):165-170.

3. Roncalli AG. Projeto SB Brasil 2010 Pesquisa Nacional de Saúde Bucal revela importante redução da cárie dentária no país. Cad Saúde Públ. 2011; 27(1):4-5.

4. Narvai PC, Frazão P, Roncalli AG, Antunes JLF. Cárie dentária no Brasil: declínio, polarização, iniqüidade e exclusão social. Rev Panam Salud Publ. 2006; 19(6). [acessado 2014 ago 14]. Disponível em: http://www.scielosp.org/ pdf/rpsp/v19n6/30519.pdf .

5. Parisotto TM, Steiner-Oliveira C, Duque C, Peres RC, Rodrigues LK, Nobre-dosSantos M. Relationship among microbiological composition and presence of dental plaque, sugar exposure, social factors and different stages of early childhood caries. Arch Oral Biol. 2010; 55(5):365-73.

6. Pinto VG. Saúde bucal coletiva. 4 ed. São Paulo: Santos, 2005.

7. Brasil. Lei $\mathrm{n}^{\circ} 11.889$ de 24 de dezembro de 2008. Regulamenta o exercício das profissões de Técnico em Saúde Bucal - 
TSB e de Auxiliar em Saúde Bucal- ASB. Diário Oficial da União 2008; 26 dez.

8. Leavell S, Clark EG. Medicina Preventiva. São Paulo: McGraw-Hill, 1976.

9. Bijella MFTB. A importância da educação em saúde bucal nos programas preventivos para crianças. J Bras Odontoped Odont Bebês. 1999; 2:12731.

10. Garbin, CAS, Rovida TAS, Peruchini LFD, Martins RJ. Conhecimento sobre saúde bucal e práticas desenvolvidas por professores do ensino fundamental e médio. RFO Passo Fundo. 2013; 18(3):321-7.

11. Brasil. Ministério da Saúde. Portaria no 4.279, de 30 de dezembro de 2010. [acessado 2014 out 07]. Disponível em: http://bvsms.saude.gov.br/bvs/saudelegis /gm/2010/prt4279_30_12_2010.html.

12. Castiel LD. O técnico e as necessidades em saúde. Cad Saúde Publica. 1985 Jan/Mar;1(1):18-24.

13. Conselho Federal de Odontologia [acessado 2014 out 08]. Disponível em: http://cfo.org.br/todas-asnoticias/programa-brasil-sorridentecompleta-10-anos/

14. Secretária de Saúde do Estado do Paraná. Saúde Bucal. [acessado 2014 out 07]. Disponível em: http://www.saude.pr.gov. br/modules/conteudo/conteudo.php?cont eudo=2896.

15. Zarifian P. Objetivo competência: por uma nova lógica. São Paulo: Atlas; 2001.

16. Narvai PC, Frazão P. Saúde Bucal no Brasil: muito além do céu da boca. Rio de Janeiro: Fiocruz, 2008.

17. Frazão P, Gonzales C, Rosa AGF. Ambiente de trabalho odontológico na perspectiva do Sistema Único de Saúde. Divulg Saúde Debate. 1995; (10):21-8.
18. Pezzato LM. O processo de formação do técnico em higiene dental e do atendente de consultório dentário, no brasil: uma história silenciada. Campinas. Dissertação [Mestrado] - Universidade Estadual de Campinas; 2001.

19. Bardin L. Análise de Conteúdo. São Paulo: Edições 70, 2011.

20. Lima JPG. Avaliação Qualitativa com cirurgiões-dentistas sobre os fatores de influência na prevenção da cárie dentária. Maringá. Dissertação [Mestrado em Clínica Integrada] - Universidade Estadual de Maringá; 2013.

21. Bonan PRF, Almeida LY, de Carvalho FMC, Brito Júnior M, Silva MS, Martelli DRB. Perfil de técnicos em higiene dental quanto à prática profissional, à educação permanente e ao trabalho em serviço público. Rev odonto ciênc. 2009; 24(2):180-5.

22. Kirschiner TC. Modernização tecnológica e formação técnico profissional no Brasil: Impasses e desafios. Instituto de Pesquisa Econômica Aplicada 1993 Mar. [acessado 2014 jun 06]. Disponível em: http://repositorio.ipea.gov.br/bitstream/1 1058/1736/1/td_0295.pdf

23. Franco MLPB, Serber A. Egressos do ensino técnico industrial no Brasil: um estudo de caso. Fund Carlos Chagas 1990. [acessado 2014 jun 23]. Disponível em: http://publicacoes.fcc.org.br/ojs/ index.php/textosfcc/article/view/2420

24. Johnson P. International profiles of dental hygiene 1987 to 2001: a 19-nation comparative study. Int Dent J 2003; 53:299-313.

25. Eaton KA, Newman H, Widstrom E. A survey of dental hygienist numbers in Canada, the European Economic area, Japan and the United States of America in 1998. Br Dent J. 2003; 195:595-8. 
26. Martins BP, Uchida TH, Terada RSS, Pascotto RC, Fujimaki M. Análise Qualitativa da percepção dos técnicos em saúde bucal sobre o desenvolvimento do papel do educador. Arch Health Invest. 2015; 4(5): 28-35.

27. Bosi MLM, Affonso KT. Cidadania, participação popular e saúde: com a palavra, os usuários da Rede Pública de Serviços. Cad Saúde Pública. 1998; 14(2):355-65.

28. Sbaraini, A. What factors influence the provision of preventive care by general dental practitioners? Br Dent J. 2012; 212(18):1-8.

29. Threfall AG, Milsom KM, Hunt CM, Tickle M, Blinkhorn AS. Exploring the content of the advice provided by general dental practitioners to help prevent caries in young children. Br Dent J. 2007; 202(9):1-4.

30. Nettleton S. Dentists and dental health education: a study of the perceptions of 28 community dentists. Community Dent Health 1989; 8:47-59.

31. Isman BA, Farrell CM. Are dental hygienists prepared to work in the changing public health environment? J Evid Base Dent Pract. 2014; 14S:183-90.

32. Wang NJ. Caries preventive methods in child dental care reported by dental hygienists, Norway, 1995 and 2004. Acta Odonl Scand. 2005; 63:330-4.

33. Bureau of Labor Statistics, US Department of Labor, Occupational Outlook Handbook, 2010-11 Edition, Dentists, Dental Hygienists. [acessado: 20.08.14] Disponível em: http://www.bls.gov/ooh/About/Projectio ns-Overview.htm

34. Jaecks KMS. Current perceptions of the role of dental hygienists in interdisciplinary collaboration. J Dent
Hyg. 2009; 83(2):84-91

35. Oliveira DG. O técnico em saúde bucal na Estratégia Saúde da Família. Catas Altas. Monografia [Curso de Especialização em Atenção Básica em Saúde da Família] - Universidade Federal de Minas Gerais; 2011.

36. Luciak-Donsberger C. Origins and benefits of dental hygiene practice in Europe. Int J Dent Hyg. 2003; 1(1):2942.

37. Narvai PC. Recursos humanos para promoção da saúde bucal: um olhar no início do século XXI. In: Kriger L. Aboprev: Promoção de Saúde Bucal. 3 ed. São Paulo: Artes Médicas; 2003. p. 475-94.

38. Abelsen B, Olsen JA. Task division between dentists and dental hygienists in Norway. Community Dent Oral Epidemiol. 2008; 36:558-66.

39. Brasil. Ministério da Saúde. Técnico em Higiene Dental e Auxiliar de Consultório Dentário: perfil de competências profissionais. Brasília; 2004. [acessado 2014 mai 20]. Disponível em: http://cfo.org.br/wpcontent/uploads/200 9/10/tecnico_higiene_dental_auxilia_co ns_dent_final.pdf

40. Frazão PA, Castellanos RA. La participación del personal auxiliar de odontología en los sistemas locales de salud. Rev Panam Salud Publica. 1999;5(2):106-115.

41. Aguiar DML. A participação do técnico em saúde bucal na estratégia saúde da família: um olhar em municípios estruturados. São Paulo. Tese [Doutorado] - Faculdade de Saúde Pública da USP; 2010.

42. Esposti CDD, Oliveira AE, Santos Neto ET, Zandonade E. O processo de trabalho do Técnico em Saúde Bucal e suas 
relações com a Equipe de Saúde Bucal na região metropolitana da Grande Vitória, Espírito Santo, Brasil. Saúde Soc. 2012; 21(2):372-385.

43. Machado MH. Trabalhadores da Saúde: Um bem público. Rev Saúde Debate. 1995;(48):54-7.
44. Francisco EM, Johnson TL, Freudenthal JJ, Louis G.Dental Hygienists' Knowledge, Attitudes and practice behaviors regarding caries risk assessment and management. J Dent Hyg. 2013 Dec; 87(6):353-61.

45. Brasil. Programa Saúde da Família. Rev Saúde Públic. 2000; 34(3):316-319.

Correspondência para:

Mitsue Fujimaki

e-mail: mfujimaki@uem.br

Departamento de Odontologia, Universidade Estadual de Maringá

Av. Mandacaru, 1550

87080-000 Maringá, Paraná 